\title{
Structural equation modelling for associated factors with dental caries among 3-5-year-old children: a cross-sectional study
}

\author{
Yuandong Qin ${ }^{\dagger}$, Rui Zhang ${ }^{\dagger}$, Bo Yuan, Ting Xu, Hong Chen, Yingming Yang ${ }^{*+}$ and Tao Hu ${ }^{*+}$
}

\begin{abstract}
Background: The aim of the current study was to explore the factors influencing dental caries among 3-5-year-old children in Sichuan Province and the interrelationship between these factors using structural equation modelling (SEM).

Methods: A cross-sectional study was conducted among 2746 3-5-year-old children in Sichuan Province. Examination of caries was conducted on all children and a questionnaire was answered by the children's caregiver. SEM alternative models were constructed to interpret the intricate relationships between socioeconomic status (SES), caregiver's oral health knowledge, attitudes, children's oral health behaviours and children's dental caries.

Result: The results showed that dental caries were significantly associated with dietary behaviours $(\beta=0.11$, $\mathrm{SE}=0.03, P=0.001, \mathrm{BC} 95 \% \mathrm{Cl}=0.05 / 0.18)$ and $\mathrm{SES}(\beta=-0.17, \mathrm{SE}=0.03, P<0.001, \mathrm{BC} 95 \% \mathrm{Cl}=-0.23 /-0.10)$ directly, While the indirect effect of SES on $\mathrm{dmft}$ is in an opposite direction $(\beta=0.08, \mathrm{SE}=0.02, \mathrm{BC} 95 \%$ $\mathrm{Cl}=0.04 / 0.12)$.

Conclusion: We found that unhealthy dietary behaviours increased the prevalence of dental caries. However, oral health knowledge and attitude failed to affect dietary behaviour in this model. This result warns that oral health education should strengthen feeding-related knowledge. Meanwhile, it also reminds that it is easier known than done. Future oral health education should focus on exploring a more effective way for the public to turn knowledge into action.
\end{abstract}

Keywords: Dental caries, KAP, Structural equation model

\section{Background}

Dental caries is one of the most severe chronic diseases among children in both developed and developing countries [1]. In 2010, dental caries in deciduous teeth affect 621 million people worldwide, which is $9 \%$ of the global population [1]. Untreated dental caries may cause severe pain and mouth infection [2], which affects children's

\footnotetext{
* Correspondence: ymyang@scu.edu.cn; hutao@scu.edu.cn

${ }^{\dagger}$ Yuandong Qin and Rui Zhang contributed equally to this work.

${ }^{\dagger}$ Tao Hu and Yingming Yang contributed equally to this paper.

State Key Laboratory of Oral Diseases \& National Clinical Research Center for

Oral Diseases \& Dept. of Preventive Dentistry, West China Hospital of

Stomatology, Sichuan University, No. 14, Sec.3, Ren Min South Road,

Chengdu, Sichuan 610041, People's Republic of China
}

school attendance and performance [3]. It is important to determine the risk factors associated with children's dental caries, and to build effective prevention strategies.

It is recognized that dental caries is caused by a multiplicity of factors, including behaviours like tooth brushing and sugar intake $[4,5]$. Conducting effective preventive behaviours relies on many factors. The knowledge-Attitude-Behaviour (KAB) model, which is developed as a health promotion model and frequently used to assess behaviour change, has been proposed as a way of explaining the role of knowledge [6]. It explains that a person's knowledge directly affects attitudes, and indirectly affects behaviours through attitudes [6]. Oral

(c) The Author(s). 2019 Open Access This article is distributed under the terms of the Creative Commons Attribution 4.0 International License (http://creativecommons.org/licenses/by/4.0/), which permits unrestricted use, distribution, and reproduction in any medium, provided you give appropriate credit to the original author(s) and the source, provide a link to the Creative Commons license, and indicate if changes were made. The Creative Commons Public Domain Dedication waiver (http://creativecommons.org/publicdomain/zero/1.0/) applies to the data made available in this article, unless otherwise stated. 
health knowledge is considered to be an essential prerequisite for health-related behaviours [7]. Furthermore, improvement in knowledge has been known to influence not only self-reported oral health-related behaviours in a favourable way, but also improve clinical parameters of oral health such as oral hygiene, gingival health and dental caries $[8,9]$. Moreover, oral health knowledge motivates positive attitudes to access information about oral health prevention and to perform oral health-related behaviours $[10,11]$. Obtaining oral health knowledge and building a positive attitude to oral health are associated with socio-economic status (SES) [11]. Meanwhile, many epidemiological studies have demonstrated an association between SES and oral health in developed countries: lower SES groups have poorer oral health than higher ones $[12,13]$.

Many factors related to dental caries have been identified by multivariate regression in previous studies, but it is not clear whether these factors influence oral health directly or indirectly. Meanwhile, dental caries is a chronic disease influenced by various aspects simultaneously [2]. Therefore, it is important to study the multidimensional factors leading to dental caries simultaneously, and to determine effective intervention measures.

Structural equation modelling (SEM) is an analytical technique for disentangling complex relationships and causal pathways when latent constructs are concerned [14]. To explore factors related to children's dental caries and the direct or indirect relationship, we proposed to build alternative models of the complex relationship between SES, knowledge, attitudes, behaviour (including dietary behaviour, tooth brushing behaviour and dental attendance) and dental caries based on theoretical framework $[11,15]$ and literature using a SEM. Model 1:We assumed that dental caries ( $\mathrm{dmft}$ ) were directly influenced by SES [16], oral health knowledge, oral health attitudes [8, 17-19], dietary behaviours [20], tooth brushing behaviours [21] and dental attendance. Simultaneously, SES, oral health knowledge and oral health attitudes directly affected dietary behaviours and tooth brushing and dental attendance [22]. Additionally, SES and oral health knowledge directly affected oral health attitudes [11], and SES directly influenced oral health knowledge [11]. Therefore, we build a direct and indirect relationship network between the relative factors and dental caries (dmft). Model 2: based on the model 1 , we assumed that oral health knowledge and attitude were directly affected by dental attendance and SES. Meanwhile, SES directly affected dental attendance, as shown in Fig. 1.

\section{Methods}

\section{Sampling and sample sizes}

Ethical approval was obtained from the Stomatological Ethics Committee of the Chinese.
Stomatological Association and the Ethics Committee of West China Hospital of Stomatology, Sichuan University (Approval No. 2014-003). A cross-sectional study was conducted among 3-5-year-old children in Sichuan Province. The sample size was calculated based on the oral disease prevalence derived from the Third National Oral Health Survey [23]. According to the formula below, the required sample size was 2472 .

$$
\mathrm{N}=\operatorname{deff} \frac{\mu^{2}(1-p)}{\varepsilon^{2} p} /(1-\text { non-responses rate })
$$

$\mathrm{N}$ is the sample size, deff means design effect (2.5), $p$ is the dental caries prevalence of 5-year-old children in the Third National Oral Health Survey because it only included 5-year-old children in the last nation oral health survey, which was $86.0 \% . \mu=1.96$ and is the level of confidence, $\varepsilon=10 \%$ and is the margin of error, the non-responses rate was $20 \%$. Hair [24] suggested that the minimum sample size for SEM was 500 for models including more than 7 constructs, and/or having fewer than three measured items. According to the estimation, we selected 2746 participants randomly to complete this study.

In this study, we obtained a representative sample by a multistage stratified cluster sampling method with selection probabilities proportional to size (PPS) [25]. Detail information regarding the sampling procedures had been published [26].

Before the study, all caregivers were provided with all details of the survey and signed informed consent were obtained.

\section{Data collect}

Data was collected through questionnaire and oral examination at the participants' kindergartens. Questionnaire study contained SES, caregiver's oral health knowledge, attitudes and children's oral health practices. Trained dentists applied the questionnaires through one-to-one interview in the classroom of kindergarten, who helped to read the questionnaire for illiteracy caregivers. The training session were hold by Chinese Stomatological Association and Peking University Hospital of stomatology in Beijing before survey. The oral examination of the children's dental caries status was performed with the aid of a mirror and a dental probe by three calibrated and accredited dentists with the assist of trained recorders, according to World Health Organization (WHO) criteria [27]. Calibration results were $>0.85$ calculated by kappa statistics. The numbers of decayed teeth and missing teeth and filled teeth were recorded based on the criteria recommended by the WHO [27]. 

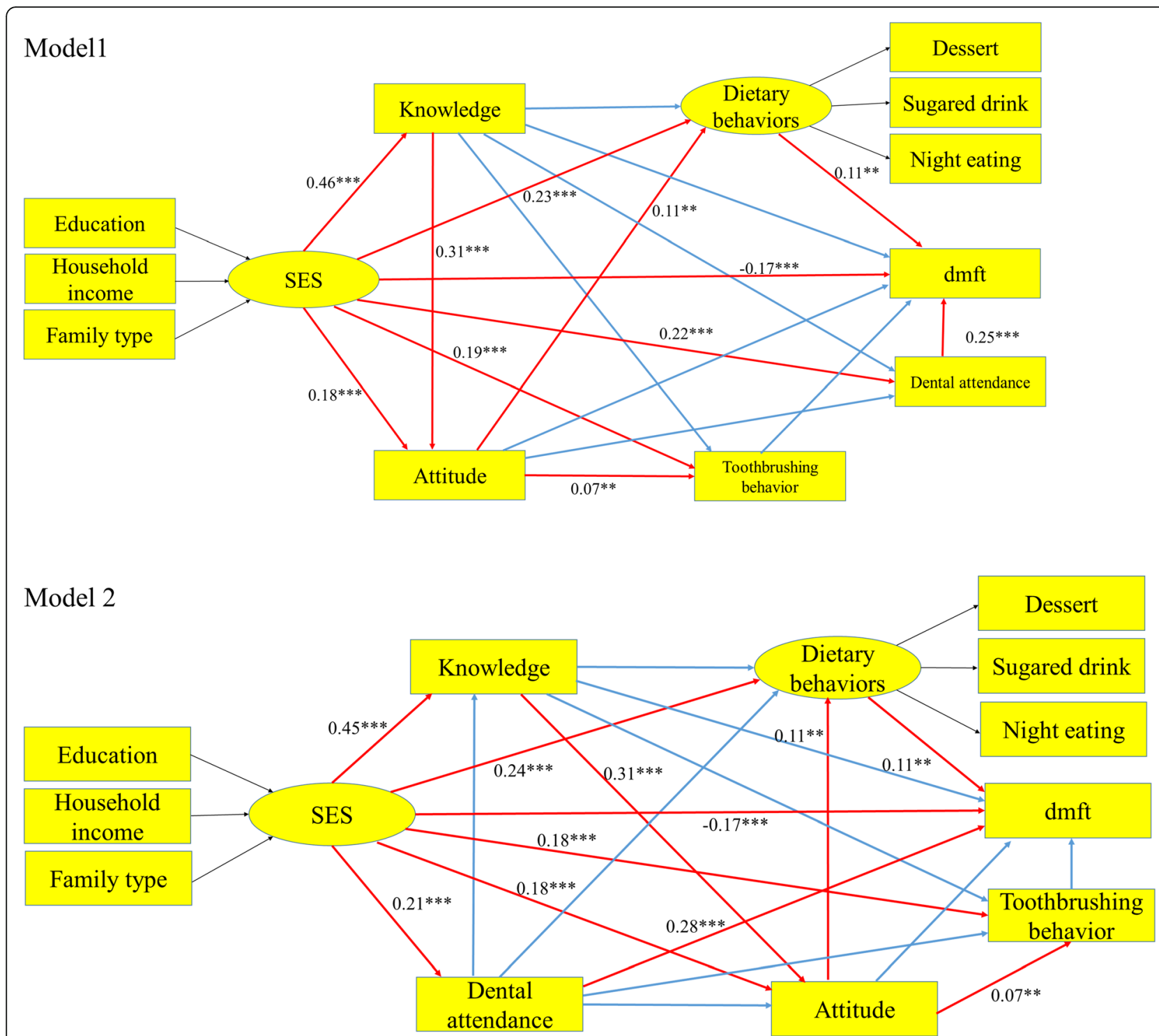

Fig. 1 Standardized path coefficients of the hypothesized model 1 and model 2. Model 1: We assumed that dental caries (dmft) were directly influenced by SES, oral health knowledge, oral health attitudes, dietary behaviours, tooth brushing behaviours and dental attendance. Simultaneously, SES, oral health knowledge and oral health attitudes directly affected dietary behaviours and tooth brushing and dental attendance. Additionally, SES and oral health knowledge directly affected oral health attitudes, and SES directly influenced oral health knowledge. Model 2: based on the model 1, we assumed that oral health knowledge and attitude were directly affected by dental attendance and SES. Meanwhile, SES directly affected dental attendance. Red solid arrows mean significant effect while blue solid arrows indicate insignificant effect (the significant for path coefficients was set at $0.05,{ }^{*} P<0.05,{ }^{* *} P<0.01,{ }^{* * *} P<0.001$ )

\section{Variables}

\section{Oral health outcome}

Participant children's oral health was evaluated by $\mathrm{dmft}$ index. The $\mathrm{dmft}$ index is commonly used for epidemiological studies in dental research [28]. Teeth or filled teeth with caries were recorded as decayed teeth (dt). Missing teeth for whatever reason in children under 9 years old were classified as missing (mt). Filled teeth without secondary caries were classified as filled ( $\mathrm{ft}$ ). The total number of $\mathrm{dt}$, $\mathrm{mt}$ and $\mathrm{ft}$. were recorded as dmft [29].

\section{Socio-economic status (SES)}

SES was measured by caregiver's education, annual household income and family type [30]. Annual household income was obtained by question that "What is your approximate total household income in the past 12 months?" Caregivers were asked to answer by an exact number. The caregivers were allowed to leave this question unanswered because income is a sensitive issue. As a result, 316 participants were excluded during analysis because of no household income information. Caregivers were required to choose their highest educational 
attainment from eight options (no formal schooling, primary school, middle school, high school, technical secondary school, junior college, university completed, postgraduate degree or above), which was recorded as the caregiver's education level. Family type was defined as non-agricultural or agricultural.

\section{Knowledge}

Caregiver's oral health knowledge was measured by eight questions [31] as shown in Table 1 and the quality of measurement was shown in Table 2. The correct answer for each question was coded as 1 , and incorrect answer or "don't know" answers were coded as 0. All eight answers were summed to create a single oral health knowledge variable ranging from 0 to 8 . Higher scores represent better oral health knowledge.

\section{Attitude}

Six questions [31] as shown in Table 1 were included as items in the caregiver's oral health attitude summary score and the quality of measurement was shown in Table 2; the answer for each question was "agree", "disagree" or "neither". Responses were coded 1 for a positive attitude and 0 for a negative attitude or neither. The final scores of oral health attitude could range from 0 to 6 ; higher scores signify a more positive oral health attitude.

\section{Dietary behaviours}

Consumption of desserts: Frequency of desserts, including cake, bread, biscuits and sweets (several times a day, every day, several times a week, once a week, several times a month, never) [32].

Sugared drink: Frequency of sugared drink, including juice, soda drinks or other soft drinks (several times a day, every day, several times a week, once a week, several times a month, never) [20].

Night eating: "your child usually has sugared drink or desserts before sleeping?" (often, sometimes, never) [33].

\section{Tooth brushing behaviours}

Tooth brushing: "Does your child brush his teeth daily?" (yes, never) [34].

\section{Dental attendance}

When was the last time your child visited a dentist?

(My child has never visited a dentist, 1 year ago, within 1 year, within half a year) [27].

\section{Gender}

Boy or Girl.

\section{Analysis}

Firstly, a descriptive analysis of all variables was done with SPSS for Windows (version 23.0; IBM Corp, Armonk,
Table 1 Questionnaire about oral health knowledge and oral health attitudes

\begin{tabular}{|c|c|c|}
\hline Number & Question & Answer \\
\hline \multicolumn{3}{|c|}{ Questions about oral health knowledge } \\
\hline 1 & $\begin{array}{l}\text { Gingival bleeding is normal } \\
\text { when toothbrushing }\end{array}$ & $\begin{array}{l}\text { No () Yes () Don't } \\
\text { know () }\end{array}$ \\
\hline 2 & $\begin{array}{l}\text { Germs are one of the reasons } \\
\text { for gingivitis }\end{array}$ & $\begin{array}{l}\text { No () Yes () Don't } \\
\text { know () }\end{array}$ \\
\hline 3 & $\begin{array}{l}\text { Toothbrushing is useless to } \\
\text { prevent gingivitis }\end{array}$ & $\begin{array}{l}\text { No () Yes () Don't } \\
\text { know () }\end{array}$ \\
\hline 4 & $\begin{array}{l}\text { Dental caries are mainly caused } \\
\text { by germs }\end{array}$ & $\begin{array}{l}\text { No () Yes () Don't } \\
\text { know () }\end{array}$ \\
\hline 5 & $\begin{array}{l}\text { Sugar consumption can lead } \\
\text { to dental caries }\end{array}$ & $\begin{array}{l}\text { No () Yes () Don't } \\
\text { know () }\end{array}$ \\
\hline 6 & $\begin{array}{l}\text { Fluoride is useless to } \\
\text { protect teeth }\end{array}$ & $\begin{array}{l}\text { No () Yes () Don't } \\
\text { know () }\end{array}$ \\
\hline 7 & $\begin{array}{l}\text { Pit and fissure sealant can } \\
\text { protect teeth }\end{array}$ & $\begin{array}{l}\text { No () Yes () Don't } \\
\text { know () }\end{array}$ \\
\hline 8 & $\begin{array}{l}\text { Oral disease could influence } \\
\text { systemic health }\end{array}$ & $\begin{array}{l}\text { No () Yes () Don't } \\
\text { know () }\end{array}$ \\
\hline \multicolumn{3}{|c|}{ Questions about oral health attitudes } \\
\hline 1 & $\begin{array}{l}\text { My oral health is very important } \\
\text { to me }\end{array}$ & $\begin{array}{l}\text { Agree () Disagree () } \\
\text { Neither () }\end{array}$ \\
\hline 2 & $\begin{array}{l}\text { Regular dental check-ups are } \\
\text { important }\end{array}$ & $\begin{array}{l}\text { Agree () Disagree () } \\
\text { Neither () }\end{array}$ \\
\hline 3 & $\begin{array}{l}\text { Teeth condition is decided at birth } \\
\text { and is not related to self-care }\end{array}$ & $\begin{array}{l}\text { Agree () Disagree () } \\
\text { Neither () }\end{array}$ \\
\hline 4 & $\begin{array}{l}\text { Self-care is important for preventing } \\
\text { dental problems }\end{array}$ & $\begin{array}{l}\text { Agree () Disagree () } \\
\text { Neither () }\end{array}$ \\
\hline 5 & $\begin{array}{l}\text { It is important to take care of } \\
\text { the first molar }\end{array}$ & $\begin{array}{l}\text { Agree () Disagree () } \\
\text { Neither () }\end{array}$ \\
\hline 6 & $\begin{array}{l}\text { Mother's oral health influences } \\
\text { children's oral health }\end{array}$ & $\begin{array}{l}\text { Agree () Disagree () } \\
\text { Neither () }\end{array}$ \\
\hline
\end{tabular}

NY). SEM was performed to test the causal relationships among observed and latent variables using Amos (SPSS plug-in software). In SEM, path analyses with latent variables (PA-LV) were applied, in which Maximum likelihood estimation and bootstrapping were used to fit functions account for the lack of multivariate normality. Two thousand bootstrap samples were re-sampled from the original data set to derive less biased standard errors and 95\% percentile confidence interval (CI) and 95\% bias-corrected confidence intervals. The model fit was evaluated by various indices used widely in SEM analysis. The model was considered feasible for the analysis only when it achieves the recommended Goodness-of-Fit

Table 2 reliability and validity of knowledge and attitude

\begin{tabular}{llll}
\hline Measure & Cronbach Alpha & KMO & $P$ of Bartlett Test \\
\hline knowledge & 0.77 & 0.79 & $<0.001$ \\
attitude & 0.73 & 0.75 & $<0.001$ \\
\hline
\end{tabular}


(GOF) measures. The chi-squared fit statistic $\left(x^{2} / \mathrm{df}\right)$, root-mean-square error of approximation (RMSEA), GOF index (GFI), normed fit index (NFI) and comparative fit index (CFI) should all be close to or better than the recommended levels when the model is acceptable [35]. In this study, the recommended GOF were used to compare models.

\section{Results}

Descriptive data of variables is shown in Table 3. The final sample was consisted of 2430 children and their caregivers. The mean annual household income is 83 thousand yuan $(\mathrm{SD}=87)$. The mean knowledge score is $4.67(\mathrm{SD}=1.52)$. The mean $\mathrm{dmft}$ is $3.29(\mathrm{SD}=4.13)$. The relationships between independent factors and children's dental caries are shown in Fig. 1. GOF measures for the models are shown (Table 4); the $x^{2} / \mathrm{df}$, RMSEA, GFI, NFI, CFI, PGFI, and PNFI of model 1 and model 2 were all close to or better than the recommended fit and model 1 is much better than model 2 . Therefore, the hypothetical model 1 was considered suitable for analysing the survey data.

The hypothetical model 1 presented in Fig. 1 has 5 major components: SES variables, oral health knowledge variables, oral health attitude variables, behavioural variables (dietary behaviours, tooth brushing behaviours and dental attendances) and dental caries variables. Figure 1 provides standardized path coefficient estimates for the proposed model 1 . Children who frequently eat desserts and sugared beverages tend to suffer from more dental caries (dmft) $\quad(\beta=0.11, \quad \mathrm{SE}=0.03, \quad P=0.001)$, which means that avoiding dessert and sugared beverage intake reduce dental caries for children. Meanwhile, tooth brushing daily did not decrease children's dental caries (dmft) $(\beta=0.01, \mathrm{SE}=0.02, P=0.73)$. At the same time, children from high SES level family are less likely to suffer from dental caries $(\beta=-0.17, \mathrm{SE}=0.03, P<0.001)$, but dental caries are not directly associated with caregiver's oral health knowledge $(\beta=-0.01, \mathrm{SE}=0.03$, $P=0.68)$ or oral health attitudes $(\beta=-0.02, \mathrm{SE}=0.02$, $P=0.41)$. Meanwhile, dental caries are positively associated with dental attendance $(\beta=0.25, \mathrm{SE}=0.02$, $P<0.001$ ), which is an interesting phenomenon in Sichuan provinces that children with more dental attendance are more likely to suffer from dental caries. Oral health behaviors, including dietary behaviors and tooth brushing behaviors and dental attendance were positively associated with SES $(\beta=0.23, \quad \mathrm{SE}=0.05$, $P<0.001$ and $\beta=0.191, \mathrm{SE}=0.03, P<0.001$ and $\beta=0.22$, $\mathrm{SE}=0.03, P<0.001$, respectively), means that caregiver with high SES level can master more oral health practices. Additionally, caregivers with positive attitude were not likely to reduce children's intake sugared food $(\beta=0.11$, $\mathrm{SE}=0.04, P=0.002$ ). Daily tooth brushing were positively
Table 3 Descriptive data of variables

\begin{tabular}{|c|c|c|}
\hline Latent variables & Observed variables & $\mathrm{n}(\%)$ or mean (SD) \\
\hline \multirow[t]{12}{*}{ SES } & Education & \\
\hline & $<$ Primary school & $267(11 \%)$ \\
\hline & Primary school & $713(29.3 \%)$ \\
\hline & Middle school & $791(32.6 \%)$ \\
\hline & High school or equivalent & $405(16.7 \%)$ \\
\hline & Technical school & $161(6.6 \%)$ \\
\hline & College graduate & $88(3.6 \%)$ \\
\hline & Advance degree & $5(0.2 \%)$ \\
\hline & Household income & $83(87)$ \\
\hline & \multicolumn{2}{|c|}{ Family type } \\
\hline & Non-agricultural & $755(31.1 \%)$ \\
\hline & Agricultural & $1675(68.9 \%)$ \\
\hline Knowledge & Knowledge scores & $4.67(1.52)$ \\
\hline Attitude & Attitude scores & $4.26(1.16)$ \\
\hline \multirow[t]{3}{*}{ Toothbrushing } & Toothbrushing & \\
\hline & Yes & $1740(71.6 \%)$ \\
\hline & Never & $690(28.4 \%)$ \\
\hline \multirow[t]{18}{*}{ dietary behaviour } & Dessert & \\
\hline & Never & $114(4.7 \%)$ \\
\hline & 1-3 times/month & $207(8.5 \%)$ \\
\hline & Once/week & $194(8.0 \%)$ \\
\hline & 2-6 times/week & $617(25.4 \%)$ \\
\hline & Once/day & 807 (33.2\%) \\
\hline & Twice/day & $491(20.2 \%)$ \\
\hline & Sugared drinks & \\
\hline & Never & $569(23.4 \%)$ \\
\hline & 1-3 times/month & $247(10.2 \%)$ \\
\hline & Once/week & $170(7.0 \%)$ \\
\hline & 2-6 times/week & $493(20.3 \%)$ \\
\hline & Once/day & $702(28.9 \%)$ \\
\hline & Twice/day & $249(10.2 \%)$ \\
\hline & Night eating & \\
\hline & Never & $353(14.5 \%)$ \\
\hline & Sometimes & $889(36.6 \%)$ \\
\hline & Often & 1188 (48.9\%) \\
\hline \multirow[t]{5}{*}{ Dental attendance } & Last time dental attendance & \\
\hline & Never & $2061(84.8 \%)$ \\
\hline & 1 year ago & $105(4.3 \%)$ \\
\hline & within 1 year & $108(4.4 \%)$ \\
\hline & within half year & $154(6.4 \%)$ \\
\hline Dental caries & $\mathrm{dmft}$ & $3.29(4.13)$ \\
\hline
\end{tabular}


Table 4 Goodness of fit measures of SEM model 1 and model 2

\begin{tabular}{lllll}
\hline Fit index & Index & $\begin{array}{l}\text { Recommend } \\
\text { levels }\end{array}$ & \multicolumn{2}{c}{$\begin{array}{l}\text { Estimate values for } \\
\text { hypothesis model }\end{array}$} \\
\cline { 4 - 5 } & & & Model 1 & Model 2 \\
\hline Absolute fit index & $\mathrm{c}^{2} / \mathrm{df}$ & $<5$ & 1.76 & 1.85 \\
& RMSEA & $<0.08$ & 0.018 & 0.019 \\
& $\mathrm{GFI}$ & $>0.9$ & 0.996 & 0.994 \\
Incremental fit indices & $\mathrm{NFI}$ & $>0.9$ & 0.97 & 0.96 \\
& $\mathrm{CFI}$ & $>0.9$ & 0.987 & 0.985 \\
\hline
\end{tabular}

$x^{2} / d f$ the chi-squared fit statistic, RMSEA root-mean-square error of approximation, GFI GOF index, NFI normed fit index, CFI comparative fit index

associated with oral health attitudes (and $\beta=0.07$, $\mathrm{SE}=0.02, P=0.003)$, but not significantly associated with oral health knowledge $(\beta=-0.01, \mathrm{SE}=0.03, P=0.577)$. Oral health attitudes were positively linked with both SES $(\beta=0.18, \mathrm{SE}=0.03, P<0.001)$ and oral health knowledge $(\beta=0.31, \mathrm{SE}=0.02, P<0.001)$. Oral health knowledge was positively associated with $\operatorname{SES}(\beta=0.46, \quad \mathrm{SE}=0.02$,
$P<0.001$ ). Table 5 and Table 6 show the corresponding regression weights and standardized regression weights of the model 1, Table 7 and Table 8 show the corresponding regression weights and standardized regression weights of the model 2.

The indirect and total effects of 5 major variables of the model were examined, and these results are presented in Table 9. The direct effect of SES on dmft is negative significantly $(\beta=-0.17, \quad \mathrm{SE}=0.03, \quad \mathrm{BC} 95 \%$ $\mathrm{CI}=-0.23 /-0.10$ ), While the indirect effect of SES on $\mathrm{dmft}$ is in an opposite direction $(\beta=0.08, \mathrm{SE}=0.02, \mathrm{BC}$ $95 \% \mathrm{CI}=0.04 / 0.12$ ), Which means that the indirect link from SES-knowledge-attitude-behaviours-dmft is broken at some point.

The total effect of SES on oral health attitude is positively significant $(\beta=0.32, \mathrm{SE}=0.02, \mathrm{BC} 95 \% \mathrm{CI}=0.27$ / 0.37), in which indirect effect of SES on oral health attitude through is a positive link $(\beta=0.14, \mathrm{SE}=0.01, \mathrm{BC}$ $95 \% \mathrm{CI}=0.12 / 0.17)$, which means the caregiver with better household SES tend to obtain positive oral health

Table 5 regression weight of the model 1

\begin{tabular}{|c|c|c|c|c|c|c|c|}
\hline & & & Estimate & SE & percentile $95 \% \mathrm{Cl}$ & Bia-corrected 95\%Cl & P \\
\hline \multicolumn{8}{|c|}{ Latent variable loadings } \\
\hline dessert & $<--$ & dietary behaviors & 1 & 1 & 1 & 1 & \\
\hline Sugared drink & $<--$ & dietary behaviors & 1.932 & 0.243 & $1.50 / 2.56$ & $1.50 / 2.56$ & $<0.001^{* * *}$ \\
\hline Night eating & $<--$ & dietary behaviors & 0.653 & 0.08 & $0.51 / 0.86$ & $0.51 / 0.87$ & $<0.001^{* * *}$ \\
\hline education & $-->$ & SES & 0.274 & 0.023 & $0.22 / 0.33$ & $0.22 / 0.33$ & $<0.001^{* * *}$ \\
\hline income & $-->$ & SES & 1 & 1 & 1 & 1 & \\
\hline Family type & $--->$ & SES & 0.05 & 0.005 & $0.04 / 0.06$ & $0.04 / 0.06$ & $<0.001^{* * *}$ \\
\hline \multicolumn{8}{|l|}{ Measured variables } \\
\hline knowledge & $<--$ & SES & 0.213 & 0.018 & $0.18 / 0.26$ & $0.18 / 0.26$ & $<0.001^{* * *}$ \\
\hline attitude & $<--$ & knowledge & 0.236 & 0.018 & $0.20 / 0.27$ & $0.20 / 0.27$ & $<0.001^{* * *}$ \\
\hline attitude & $<--$ & SES & 0.064 & 0.011 & $0.04 / 0.09$ & $0.05 / 0.09$ & $<0.001^{* * *}$ \\
\hline dietary behaviors & $<--$ & attitude & 0.046 & 0.015 & $0.02 / 0.08$ & $0.02 / 0.08$ & $0.002^{* *}$ \\
\hline dietary behaviors & $<--$ & knowledge & -0.002 & 0.012 & $-0.03 / 0.03$ & $-0.03 / 0.02$ & 0.885 \\
\hline Toothbrushing & $<--$ & knowledge & -0.004 & 0.008 & $-0.02 / 0.01$ & $-0.02 / 0.01$ & 0.577 \\
\hline toothbrushing & $<---$ & attitude & 0.026 & 0.009 & $0.01 / 0.04$ & $0.01 / 0.04$ & $0.003^{* *}$ \\
\hline toothbrushing & $<--$ & SES & 0.026 & 0.005 & $0.02 / 0.04$ & $0.02 / 0.04$ & $<0.001^{* * *}$ \\
\hline dietary behaviors & $<--$ & SES & 0.034 & 0.008 & $0.02 / 0.05$ & $0.02 / 0.05$ & $<0.001^{* * *}$ \\
\hline Dental attendance & $<--$ & SES & 0.055 & 0.009 & $0.04 / 0.07$ & $0.04 / 0.08$ & $<0.001^{* * *}$ \\
\hline Dental attendance & $<--$ & knowledge & -0.007 & 0.014 & $-0.03 / 0.02$ & $-0.04 / 0.02$ & 0.635 \\
\hline Dental attendance & $<--$ & attitude & 0.014 & 0.016 & $-0.01 / 0.04$ & $-0.01 / 0.04$ & 0.389 \\
\hline $\mathrm{dmft}$ & $<--$ & SES & -0.208 & 0.046 & $-0.31 /-0.13$ & $-0.31 /-0.13$ & $<0.001^{* * *}$ \\
\hline dmft & $<--$ & knowledge & -0.028 & 0.068 & $-0.17 / 0.11$ & $-0.17 / 0.11$ & 0.68 \\
\hline $\mathrm{dmft}$ & $<--$ & attitude & -0.065 & 0.08 & $-0.22 / 0.10$ & $-0.23 / 0.08$ & 0.41 \\
\hline $\mathrm{dmft}$ & $<--$ & dietary behaviors & 0.945 & 0.295 & $0.36 / 1.59$ & $0.38 / 1.61$ & $0.001^{* *}$ \\
\hline dmft & $<--$ & toothbrushing & 0.066 & 0.188 & $-0.31 / 0.45$ & $-0.31 / 0.46$ & 0.73 \\
\hline $\mathrm{dmft}$ & $<--$ & dental attendance & 1.253 & 0.102 & $1.02 / 1.50$ & $1.01 / 1.49$ & $<0.001^{* * *}$ \\
\hline
\end{tabular}


Table 6 standardized regression weight of the model 1

\begin{tabular}{|c|c|c|c|c|c|c|c|}
\hline & & & Estimate & SE & percentile $95 \% \mathrm{Cl}$ & Bia-corrected 95\%Cl & $P$ \\
\hline \multicolumn{8}{|l|}{ Latent variable loadings } \\
\hline dessert & $<--$ & dietary behaviors & 0.35 & 0.03 & $0.29 / 0.42$ & $0.29 / 0.42$ & $<0.001^{* * *}$ \\
\hline sugared drink & $<--$ & dietary behaviors & 0.53 & 0.04 & $0.46 / 0.61$ & $0.45 / 0.61$ & $<0.001^{* * *}$ \\
\hline night eating & $<--$ & dietary behaviors & 0.44 & 0.03 & $0.37 / 0.50$ & $0.37 / 0.50$ & $<0.001^{* * *}$ \\
\hline education & $--->$ & SES & 0.73 & 0.03 & 0.68/0.79 & $0.68 / 0.79$ & $<0.001^{* * *}$ \\
\hline income & $--->$ & SES & 0.38 & 0.03 & $0.33 / 0.44$ & $0.32 / 0.44$ & $<0.001^{* * *}$ \\
\hline Family type & $--->$ & SES & 0.35 & 0.03 & $0.30 / 0.40$ & $0.31 / 0.40$ & $<0.001^{* * *}$ \\
\hline \multicolumn{8}{|l|}{ Measured variables } \\
\hline knowledge & $<--$ & SES & 0.46 & 0.02 & $0.41 / 0.50$ & $0.41 / 0.50$ & $<0.001^{* * *}$ \\
\hline attitude & $<--$ & knowledge & 0.31 & 0.02 & $0.26 / 0.36$ & $0.26 / 0.36$ & $<0.001^{* * *}$ \\
\hline attitude & $<--$ & SES & 0.18 & 0.03 & $0.13 / 0.23$ & $0.13 / 0.23$ & $<0.001^{* * *}$ \\
\hline dietary behaviors & $<--$ & attitude & 0.11 & 0.04 & $0.04 / 0.18$ & $0.04 / 0.18$ & $0.002^{* *}$ \\
\hline dietary behaviors & $<--$ & knowledge & -0.01 & 0.04 & $-0.08 / 0.07$ & $-0.08 / 0.08$ & 0.885 \\
\hline toothbrushing & $<---$ & knowledge & -0.01 & 0.03 & $-0.06 / 0.03$ & $-0.06 / 0.03$ & 0.577 \\
\hline toothbrushing & $<--$ & attitude & 0.07 & 0.02 & $0.03 / 0.11$ & $0.02 / 0.11$ & $0.003^{* *}$ \\
\hline toothbrushing & $<--$ & SES & 0.19 & 0.03 & $0.13 / 0.25$ & $0.13 / 0.25$ & $<0.001^{* * *}$ \\
\hline dietary behaviors & $<--$ & SES & 0.23 & 0.05 & $0.14 / 0.32$ & $0.14 / 0.32$ & $<0.001^{* * *}$ \\
\hline Dental attendance & $<--$ & SES & 0.22 & 0.03 & $0.15 / 0.28$ & $0.15 / 0.29$ & $<0.001^{* * *}$ \\
\hline Dental attendance & $<---$ & knowledge & -0.01 & 0.03 & $-0.06 / 0.04$ & $-0.06 / 0.04$ & 0.635 \\
\hline Dental attendance & $<--$ & attitude & 0.02 & 0.02 & $-0.02 / 0.06$ & $-0.02 / 0.06$ & 0.389 \\
\hline $\mathrm{dmft}$ & $<--$ & SES & -0.17 & 0.03 & $-0.23 /-0.10$ & $-0.23 /-0.10$ & $<0.001^{* * *}$ \\
\hline $\mathrm{dmft}$ & $<--$ & knowledge & -0.01 & 0.03 & $-0.06 / 0.04$ & $-0.06 / 0.04$ & 0.68 \\
\hline dmft & $<--$ & attitude & -0.02 & 0.02 & $-0.06 / 0.03$ & $-0.06 / 0.02$ & 0.41 \\
\hline dmft & $<--$ & Dietary behaviors & 0.11 & 0.03 & $0.04 / 0.17$ & $0.05 / 0.18$ & $0.001^{* *}$ \\
\hline $\mathrm{dmft}$ & $<--$ & toothbrushing & 0.01 & 0.02 & $-0.03 / 0.05$ & $-0.03 / 0.05$ & 0.73 \\
\hline $\mathrm{dmft}$ & $<--$ & dental attendance & 0.25 & 0.02 & $0.21 / 0.30$ & $0.21 / 0.30$ & $* * *$ \\
\hline
\end{tabular}

attitude. The total association of SES to dietary behaviors is opposite of the hypothesized direction $(\beta=0.27$, $\mathrm{SE}=0.04, \mathrm{BC} 95 \% \mathrm{CI}=0.19 / 0.35)$ as well as the indirect effect $(\beta=0.03, \mathrm{SE}=0.02, \mathrm{BC} 95 \% \mathrm{CI}=0.00 / 0.07)$ and direct effect $(\beta=0.23, \mathrm{SE}=0.05, \mathrm{BC} 95 \% \mathrm{CI}=0.14$ / 0.32). Which means that caregivers with better SES were not more likely to avoid sugared beverage intake. SES effect on tooth brushing behaviours positive directly $(\beta=0.19, \mathrm{SE}=0.03, \mathrm{BC} 95 \% \mathrm{CI}=0.13 / 0.25)$ but not significant for indirect $(\beta=0.02, \mathrm{SE}=0.01, \mathrm{BC} 95 \%$ $\mathrm{CI}=-0.01 / 0.04)$. So far we narrow the broken point into oral health knowledge-oral health attitude-behaviours. The total effect of knowledge on both dietary behaviours and toothbrushing behaviours are not significant $(\beta=0.03, \mathrm{SE}=0.04, \mathrm{BC} 95 \% \mathrm{CI}=-0.04 / 0.11$ and $\beta=$ $0.02, \mathrm{SE}=0.03, \mathrm{BC} 95 \% \mathrm{CI}=-0.04 / 0.06$, respectively). Given that direct effect of attitude on dietary behaviours is opposite to the hypothesis direction mentioned above. The link oral health knowledge and attitude with oral health behaviours is separation, which lead to the indirect effect of SES on dmft is not significant.

\section{Discussion}

This study provides comprehensive information about factors associated with dental caries in 3-5 years old children in Sichuan Province. Dietary behaviours were also directly associated with dental caries. The high caries experience was more commonly seen in children who often pick sugared beverage and dessert, and who have night eating behaviour; previous study was also in accordance with that [36]. Therefore, warning labels pertaining sugar intake is necessary. And, it is also important for clinicians to analysis children' dietary behaviours and give advice about dietary in treatment plans.

Here SES is directly associated with children's dmft, children from higher income and education families had a significantly lower chance to suffer dental caries. These findings were consistent with previous studies [37]. 
Table 7 Regression weight of the model 2

\begin{tabular}{|c|c|c|c|c|c|c|c|}
\hline & & & Estimate & SE & percentile $95 \% \mathrm{Cl}$ & Bia-corrected 95\%Cl & $P$ \\
\hline \multicolumn{8}{|l|}{ Latent variable loadings } \\
\hline dessert & $<--$ & dietary behaviors & 1 & & 1 & 1 & \\
\hline Sugared drink & $<--$ & dietary behaviors & 1.94 & 0.25 & $1.51 / 2.09$ & $1.50 / 2.58$ & $<0.001^{* * *}$ \\
\hline Night eating & $<---$ & dietary behaviors & 0.65 & 0.08 & $0.50 / 0.86$ & $0.50 / 0.86$ & $<0.001^{* * *}$ \\
\hline education & $<---$ & SES & 1 & & 1 & 1 & \\
\hline income & $<--$ & SES & 3.62 & 0.3 & $2.99 / 4.41$ & $3.00 / 4.43$ & $<0.001^{* * *}$ \\
\hline Family type & $<--$ & SES & 0.18 & 0.02 & $0.15 / 0.22$ & $0.15 / 0.24$ & $<0.001^{* * *}$ \\
\hline \multicolumn{8}{|l|}{ Measured variables } \\
\hline dental attendance & $<--$ & SES & 0.08 & 0.01 & $0.06 / 0.11$ & $0.06 / 0.11$ & $<0.001^{* * *}$ \\
\hline knowledge & $<--$ & SES & 0.77 & 0.06 & $0.65 / 0.89$ & $0.65 / 0.90$ & $<0.001^{* * *}$ \\
\hline knowledge & $<--$ & dental attendance & 0.06 & 0.09 & $-0.12 / 0.22$ & $-0.12 / 0.21$ & 0.54 \\
\hline attitude & $<--$ & knowledge & 0.24 & 0.02 & $0.20 / 0.27$ & $0.20 / 0.27$ & $<0.001^{* * *}$ \\
\hline attitude & $<--$ & SES & 0.23 & 0.04 & $0.15 / 0.30$ & $0.16 / 0.31$ & $<0.001^{* * *}$ \\
\hline attitude & $<--$ & dental attendance & 0.06 & 0.06 & $-0.05 / 0.16$ & $-0.05 / 0.16$ & 0.36 \\
\hline dietary behaviors & $<---$ & attitude & 0.05 & 0.02 & $0.02 / 0.08$ & $0.02 / 0.08$ & $0.003^{* *}$ \\
\hline toothbrushing & $<--$ & attitude & 0.03 & 0.01 & $0.01 / 0.04$ & $0.01 / 0.04$ & $0.002^{* *}$ \\
\hline toothbrushing & $<--$ & SES & 0.09 & 0.02 & $0.06 / 0.12$ & $0.06 / 0.12$ & $<0.001^{* * *}$ \\
\hline dietary behaviors & $<--$ & SES & 0.13 & 0.03 & $0.07 / 0.19$ & $0.07 / 0.19$ & $<0.001^{* * *}$ \\
\hline dietary behaviors & $<--$ & dental attendance & -0.03 & 0.04 & $-0.11 / 0.07$ & $-0.11 / 0.07$ & 0.58 \\
\hline toothbrushing & $<--$ & dental attendance & 0.04 & 0.03 & $-0.01 / 0.09$ & $-0.01 / 0.09$ & 0.13 \\
\hline dietary behaviors & $<--$ & knowledge & 0 & 0.01 & $-0.03 / 0.02$ & $-0.03 / 0.02$ & 0.88 \\
\hline toothbrushing & $<---$ & knowledge & 0 & 0 & $-0.02 / 0.01$ & $-0.02 / 0.01$ & 0.64 \\
\hline $\mathrm{dmft}$ & $<--$ & attitude & -0.07 & 0.08 & $-0.22 / 0.09$ & $-0.23 / 0.08$ & 0.4 \\
\hline $\mathrm{dmft}$ & $<---$ & dietary behaviors & 0.93 & 0.29 & $0.38 / 1.57$ & $0.40 / 1.59$ & $0.001^{* *}$ \\
\hline $\mathrm{dmft}$ & $<--$ & toothbrushing & 0.04 & 0.19 & $-0.34 / 0.43$ & $-0.33 / 0.43$ & 0.83 \\
\hline $\mathrm{dmft}$ & $<--$ & dental attendance & 3.22 & 0.24 & $2.66 / 3.78$ & $2.63 / 3.76$ & $<0.001^{* * *}$ \\
\hline $\mathrm{dmft}$ & $<--$ & SES & -0.76 & 0.17 & $-1.13 /-0.45$ & $-1.14 /-0.46$ & $<0.001^{* * *}$ \\
\hline $\mathrm{dmft}$ & $<---$ & knowledge & -0.04 & 0.07 & $-0.18 / 0.09$ & $-0.18 / 0.09$ & 0.51 \\
\hline
\end{tabular}

However, SES is positively associated with dietary behaviours, the potential reason to interpret this phenomenon is that family with high income and education do not realize the risk of sugared beverage and dessert intake. Parents need to have better oral health education about feeding.

Both SES and attitude are positively linked with toothbrushing, but toothbrushing isn't significant with the prevalence of dental caries. However toothbrushing is the recommended oral hygiene methods to prevent dental caries have been widely proven. This finding indicates that children in Sichuan provinces don't brush their teeth with rightly method. In our study, only $57.7 \%$ of children among those who brush their teeth frequently have ever brushed teeth under a caregiver's supervision, which cannot guarantee the effectiveness of toothbrushing. Proper technique of brushing teeth such as Bass Method should be widely publicize [38]. Parents should remind their child to brush daily and help their child to brush their teeth before the recommended age [39].

Dental caries were significantly associated with oral health behaviours and SES directly. However, the indirect link through traditional oral health education link SES-knowledge-attitude-behaviour pathway did not influence dmft. Figure out the vulnerable spot can help the planning and evaluation of the oral health promotion program for children 3-5 years old in Sichuan province. In this study, SES is positively associated with oral health knowledge and attitude. Generally speaking, people with high SES will have much more opportunity to access information about health, which is consistent with previous study [11]. We noticed that SES influence attitude 
Table 8 standardized regression weight of the model 2

\begin{tabular}{|c|c|c|c|c|c|c|c|}
\hline & & & Estimate & SE & percentile $95 \% \mathrm{Cl}$ & Bia-corrected 95\%Cl & $P$ \\
\hline \multicolumn{8}{|l|}{ Latent variable loadings } \\
\hline dessert & $<--$ & dietary behaviors & 0.351 & 0.034 & $0.28 / 0.42$ & $0.28 / 0.42$ & $<0.001^{* * *}$ \\
\hline Sugared drink & $<---$ & dietary behaviors & 0.532 & 0.041 & $0.46 / 0.62$ & $0.46 / 0.61$ & $<0.001^{* * *}$ \\
\hline Night eating & $<--$ & dietary behaviors & 0.433 & 0.034 & $0.37 / 0.50$ & $0.37 / 0.50$ & $<0.001^{* * *}$ \\
\hline education & $<---$ & SES & 0.734 & 0.03 & $0.68 / 0.80$ & $0.68 / 0.80$ & $<0.001^{* * *}$ \\
\hline income & $<--$ & SES & 0.353 & 0.03 & $0.30 / 0.40$ & $0.30 / 0.40$ & $<0.001^{* * *}$ \\
\hline Family type & $<---$ & SES & 0.375 & 0.03 & $0.33 / 0.44$ & $0.32 / 0.43$ & $<0.001^{* * *}$ \\
\hline \multicolumn{8}{|l|}{ Measured variables } \\
\hline dental attendance & $<--$ & SES & 0.212 & 0.027 & $0.16 / 0.27$ & $0.16 / 0.27$ & $<0.001^{* * *}$ \\
\hline knowledge & $<---$ & SES & 0.453 & 0.025 & $0.40 / 0.50$ & $0.40 / 0.50$ & $<0.001^{* * *}$ \\
\hline knowledge & $<--$ & dental attendance & 0.013 & 0.019 & $-0.03 / 0.05$ & $-0.03 / 0.05$ & 0.54 \\
\hline attitude & $<---$ & knowledge & 0.31 & 0.023 & $0.27 / 0.36$ & $0.27 / 0.36$ & $<0.001^{* * *}$ \\
\hline attitude & $<--$ & SES & 0.177 & 0.028 & $0.12 / 0.23$ & $0.12 / 0.23$ & $<0.001^{* * *}$ \\
\hline attitude & $<---$ & dental attendance & 0.018 & 0.017 & $-0.02 / 0.05$ & $-0.02 / 0.05$ & 0.36 \\
\hline dietary behaviors & $<---$ & attitude & 0.111 & 0.035 & $0.04 / 0.18$ & $0.04 / 0.18$ & $0.003^{* *}$ \\
\hline toothbrushing & $<---$ & attitude & 0.068 & 0.023 & $0.03 / 0.11$ & $0.03 / 0.11$ & $0.002^{* *}$ \\
\hline toothbrushing & $<--$ & SES & 0.175 & 0.031 & $0.12 / 0.24$ & $0.12 / 0.24$ & $<0.001^{* * *}$ \\
\hline dietary behaviors & $<--$ & SES & 0.24 & 0.051 & $0.14 / 0.36$ & $0.14 / 0.34$ & $<0.001^{* * *}$ \\
\hline dietary behaviors & $<--$ & dental attendance & -0.018 & 0.034 & $-0.08 / 0.05$ & $-0.08 / 0.05$ & 0.58 \\
\hline toothbrushing & $<--$ & dental attendance & 0.031 & 0.02 & $-0.01 / 0.07$ & $-0.01 / 0.07$ & 0.13 \\
\hline dietary behaviors & $<--$ & knowledge & -0.006 & 0.04 & $-0.08 / 0.08$ & $-0.08 / 0.08$ & 0.88 \\
\hline toothbrushing & $<--$ & knowledge & -0.012 & 0.025 & $-0.06 / 0.04$ & $-0.06 / 0.04$ & 0.64 \\
\hline $\mathrm{dmft}$ & $<--$ & attitude & -0.019 & 0.022 & $-0.06 / 0.03$ & $-0.07 / 0.02$ & 0.4 \\
\hline $\mathrm{dmft}$ & $<---$ & dietary behaviors & 0.108 & 0.033 & $0.04 / 0.17$ & $0.05 / 0.17$ & $0.001^{* *}$ \\
\hline $\mathrm{dmft}$ & $<--$ & toothbrushing & 0.004 & 0.021 & $-0.04 / 0.05$ & $-0.04 / 0.05$ & 0.83 \\
\hline $\mathrm{dmft}$ & $<---$ & dental attendance & 0.28 & 0.024 & $0.23 / 0.33$ & $0.23 / 0.33$ & $<0.001^{* * *}$ \\
\hline $\mathrm{dmft}$ & $<--$ & SES & -0.166 & 0.034 & $-0.23 /-0.10$ & $-0.23 /-0.10$ & $<0.001^{* * *}$ \\
\hline $\mathrm{dmft}$ & $<---$ & knowledge & -0.016 & 0.025 & $-0.07 / 0.03$ & $-0.07 / 0.03$ & 0.51 \\
\hline
\end{tabular}

through knowledge, which means our oral health knowledge education is effective. A study in Guangzhou in 2014 [11] found that caregivers with better SES were equipped with better oral health knowledge and attitudes. The link from dietary behaviours to $\mathrm{dmft}$ is also significant. Therefore, vulnerable spot in the whole SES-knowledge-attitude-behaviours-dmft should be located in the knowledge-attitude-behaviours part. The indirect effect of SES on dietary behaviours through oral health knowledge and attitude is opposite with hypothesis association. The indirect effect of SES on toothbrushing through oral health knowledge and attitude is not significant. The total effect of knowledge on oral health behaviours (dietary behaviours and toothbrushing behaviours) isn't significant. So we found that the association between oral health knowledge, attitude and oral health behaviours is separated. In the present study, oral health behaviours were not directly or indirectly associated with oral health knowledge. This is consistent with findings from a Singapore study that questioned the theoretical assumption of the knowledge-attitude-practice (KAP) links and the notion that health improvements are achievable through professional health education [15]. This finding recommends that specialists should rethink about health education contents. Health educators should educate and motivate caregivers with specific advice and information triggering corresponding practices instead of giving general oral health knowledge. A layperson does not necessarily have knowledge about how germs causing dental caries and how fluoride prevents tooth decay.

Dental clinic attendance was positively related to dental caries, which indicated abnormal phenomenon in Sichuan provinces. Children were more likely to use dental care services for tooth pain instead of regular checkups, which 
Table 9 Total effect, direct effect and indirect of the variables of model 1

\begin{tabular}{|c|c|c|c|c|c|c|c|}
\hline & & & & estimate & SE & Bia-corrected 95\%Cl & percentile $95 \% \mathrm{Cl}$ \\
\hline \multirow[t]{3}{*}{ attitude } & $<--$ & SES & Total & 0.32 & 0.02 & $0.27 / 0.37$ & $0.27 / 0.37$ \\
\hline & & & indirect & 0.14 & 0.01 & $0.12 / 0.17$ & $0.12 / 0.17$ \\
\hline & & & direct & 0.18 & 0.03 & $0.13 / 0.23$ & $0.13 / 0.23$ \\
\hline \multirow[t]{3}{*}{ toothbrushing } & $<---$ & SES & Total & 0.20 & 0.03 & $0.16 / 0.25$ & $0.16 / 0.25$ \\
\hline & & & indirect & 0.02 & 0.01 & $-0.01 / 0.04$ & $-0.01 / 0.04$ \\
\hline & & & direct & 0.19 & 0.03 & $0.13 / 0.25$ & $0.13 / 0.25$ \\
\hline \multirow[t]{3}{*}{ dietary behaviors } & $<--$ & SES & Total & 0.27 & 0.04 & $0.19 / 0.35$ & $0.19 / 0.34$ \\
\hline & & & indirect & 0.03 & 0.02 & $0.00 / 0.07$ & $0.00 / 0.07$ \\
\hline & & & direct & 0.23 & 0.05 & $0.14 / 0.32$ & $0.14 / 0.32$ \\
\hline \multirow[t]{3}{*}{ dental attendance } & $<---$ & SES & Total & 0.22 & 0.03 & $0.16 / 0.27$ & $0.16 / 0.27$ \\
\hline & & & indirect & 0 & 0.01 & $-0.02 / 0.02$ & $-0.02 / 0.02$ \\
\hline & & & direct & 0.22 & 0.03 & $0.15 / 0.29$ & $0.15 / 0.28$ \\
\hline \multirow[t]{3}{*}{$\mathrm{dmft}$} & $<--$ & SES & Total & -0.09 & 0.03 & $-0.14 /-0.04$ & $-0.14 /-0.04$ \\
\hline & & & indirect & 0.08 & 0.02 & $0.04 / 0.12$ & $0.04 / 0.12$ \\
\hline & & & direct & -0.17 & 0.03 & $-0.23 /-0.10$ & $-0.21 /-0.07$ \\
\hline toothbrushing & $<---$ & knowledge & Total & 0.02 & 0.03 & $-0.04 / 0.06$ & $-0.14 /-0.04$ \\
\hline dietary behaviors & $<---$ & knowledge & Total & 0.03 & 0.04 & $-0.04 / 0.11$ & $-0.05 / 0.11$ \\
\hline $\mathrm{dmft}$ & $<---$ & knowledge & Total & -0.01 & 0.03 & $-0.07 / 0.03$ & $-0.07 / 0.04$ \\
\hline $\mathrm{dmft}$ & $<--$ & attitude & Total & -0.01 & 0.02 & $-0.05 / 0.04$ & $-0.04 / 0.04$ \\
\hline
\end{tabular}

have been reported in previous studies in another region [40]. Therefore, children using dental care services were more likely to experience dental caries. It is necessary to emphasize the important of regular check-up and oral preventive care.

The greatest strength of our study lies in the integration of various factors with untreated dental caries by SEM, in which superior and multiple regression modelling can show direct relationships between one oral health outcome and various risk factors. SEM was first used in the field of social sciences and has become popular in dental sciences [40-42]. SEM is a multivariate statistical method able to evaluate a network of relationships between observed and latent variables, and measure the overall model fit [43]. Model fit to the data was assessed with various indices used widely in SEM analysis. To our knowledge, this was the first SEM model applied to data from Sichuan Province. The second strength is that our sample size is sufficiently large enough to represent the entire population of Sichuan Province.

This study has limitations. First, dental caries is caused by multiple factors, which contain physical, biological, environmental, behaviour and lifestyle-related factors such as high numbers of cariogenic bacteria, inadequate salivary flow, insufficient fluoride exposure, poor oral hygiene, inappropriate methods of feeding infants and poverty [2]. We only included some of these risk factors in our study. Second, this study was a cross-sectional study; however, dental caries is a chronic and progressive disease better suited to longitudinal research.

\section{Conclusion}

SES and dietary behaviour variables play a crucial role in explaining dental caries outcomes, children from high level SES family were more likely to suffer from dental caries.

Generally, good oral health knowledge and positive oral health attitude can improve oral health, however, oral health knowledge and attitude failed to affect dietary behaviour in this model, meanwhile we find that unhealthy dietary behaviour can lead to an increase in caries. These results demonstrate that the knowledge of healthy feeding is not propagated enough in Sichuan province.

SES affects oral health knowledge and attitude, but oral health knowledge and attitude failed to affect dietary behaviour in our research. This founding warns that it is easier than done. Future oral health education should focus on finding a more effective way for the public to turn knowledge into action. A policy for dental caries prevention should focus on effective oral health education and triggering corresponding protective oral health behaviours.

\section{Abbreviations}

CFI: Comparative fit index; GFI: GOF index; GOF: Goodness-of-Fit; NFI: Normed fit index; PA-LV: Path analyses with latent variables;

PPS: Probabilities proportional to size; RMSEA: Root-mean-square error of 
approximation; SEM: Structural equation modelling; SES: Socio-economic status; $x^{2} / d f:$ Chi-squared fit statistic

\section{Acknowledgements}

The authors thank the technical assistance provided by the Sichuan Centre for Disease Control and Prevention, survey team from the West China Hospital of Stomatology, Sichuan University, P. R. China.

\section{Funding}

This survey was funded by grants from Special Project Funds of Chengdu Science and Technology Bureau (Grant No. 2015-HM01-00501-SF) and Sichuan Provincial Natural Science Foundation of China (Grant No. 2018SZ0139), the funders of the study had no role in the design of the study and collection, analysis, and interpretation of data and in writing the manuscript.

\section{Availability of data and materials}

The datasets used and analyzed during the current study are available from the corresponding author on reasonable request.

\section{Authors' contributions}

QYD, ZR: Conception and design of the work, doing a computerized literature search, acquisition of data, or analysis and interpretation of data. QYD, ZR, YYM, HT: Drafting the article or revising it critically for important intellectual content. QYD, ZR, YB, XT, CH, YYM, HT: Agreement to be accountable for all aspects of the work. YB, YYM, HT: Conception and design of the work. XT: Collect the data. $\mathrm{CH}$ : Provide assistant with data analysis. All authors have made substantive contribution to this study and/or manuscript, and all have reviewed the final paper prior to its submission. All authors read and approved the final manuscript.

\section{Ethics approval and consent to participate}

Ethical approvals were obtained from the Stomatological Ethics Committee of the Chinese Stomatological Association and the Ethics Committee of West China Hospital of Stomatology, Sichuan University (Approval No. 2014-003), All parents were required to sign an informed consent form, the informed consent form contains the survey content about parents and children.

\section{Competing interests}

The authors declare that they have no competing interests.

\section{Publisher's Note}

Springer Nature remains neutral with regard to jurisdictional claims in published maps and institutional affiliations.

\section{Received: 5 October 2018 Accepted: 15 May 2019} Published online: 06 June 2019

\section{References}

1. Kassebaum NJ, Bernabe E, Dahiya M, Bhandari B, Murray CJ, Marcenes W. Global burden of untreated caries: a systematic review and metaregression. J Dent Res. 2015;94(5):650-8.

2. Selwitz RH, Ismail Al, Pitts NB. Dental caries. Lancet. 2007;369(9555):51-9.

3. Jackson SL, Vann WF Jr, Kotch JB, Pahel BT, Lee JY. Impact of poor oral health on children's school attendance and performance. Am J Public Health. 2011;101(10):1900-6.

4. Macpherson LM, Anopa Y, Conway DI, McMahon AD. National supervised toothbrushing program and dental decay in Scotland. J Dent Res. 2013; 92(2):109-13.

5. Moynihan PJ, Kelly SA. Effect on caries of restricting sugars intake: systematic review to inform WHO guidelines. J Dent Res. 2014;93(1):8-18.

6. Yi Q, Hohashi N. Comparison of perceptions of domestic elder abuse among healthcare workers based on the Knowledge-Attitude-Behavior (KAB) model. PLoS One. 2018;13(11):e0206640.

7. Al-Ansari J, Honkala E, Honkala S. Oral health knowledge and behavior among male health sciences college students in Kuwait. BMC Oral Health. 2003;3(1):2.

8. Haque SE, Rahman M, Itsuko K, Mutahara M, Kayako S, Tsutsumi A, Islam MJ, Mostofa MG. Effect of a school-based oral health education in preventing untreated dental caries and increasing knowledge, attitude, and practices among adolescents in Bangladesh. BMC Oral Health. 2016;16:44.
9. Brennan D, Spencer J, Roberts-Thomson K. Dental knowledge and oral health among middle-aged adults. Aust N Z J Public Health. 2010; 34(5):472-5.

10. Rad M, Shahravan A, Haghdoost AA. Oral health knowledge, attitude, and practice in 12-year-old schoolchildren in Iran. J Int Soc Prev Community Dent. 2015;5(5):419-24.

11. Qiu RM, Lo EC, Zhi QH, Zhou Y, Tao Y, Lin HC. Factors related to children's caries: a structural equation modeling approach. BMC Public Health. 2014;14:1071.

12. Watt RG. From victim blaming to upstream action: tackling the social determinants of oral health inequalities. Community Dent Oral Epidemiol. 2007;35(1):1-11.

13. Petersen PE, Kwan S. Equity, social determinants and public health programmes--the case of oral health. Community Dent Oral Epidemiol. 2011;39(6):481-7.

14. Newton JT, Bower EJ. The social determinants of oral health: new approaches to conceptualizing and researching complex causal networks. Community Dent Oral Epidemiol. 2005;33(1):25-34.

15. Gao XL, Hsu CY, Xu YC, Loh T, Koh D, Hwarng HB. Behavioral pathways explaining oral health disparity in children. J Dent Res. 2010;89(9):985-90.

16. Andre Kramer AC, Petzold M, Hakeberg M, Ostberg AL. Multiple Socioeconomic Factors and Dental Caries in Swedish Children and Adolescents. Caries Res. 2018;52(1-2):42-50.

17. Hamilton ME, Coulby WM. Oral health knowledge and habits of senior elementary school students. J Public Health Dent. 1991;51(4):212-9.

18. Gupta T, Sequeira P, Acharya S. Oral health knowledge, attitude and practices of a 15-year-old adolescent population in Southern India and their social determinants. Oral Health Prev Dent. 2012;10(4):345-54.

19. Harikiran AG, Pallavi SK, Hariprakash S, Nagesh KS. Oral health-related KAP among 11- to 12-year-old school children in a government-aided missionary school of Bangalore city. Indian J Dent Res. 2008;19(3):236-42.

20. Sheiham A, James WP. Diet and Dental Caries: The Pivotal Role of Free Sugars Reemphasized. J Dent Res. 2015;94(10):1341-7.

21. Sun HB, Zhang W, Zhou XB. Risk Factors associated with Early Childhood Caries. Chin J Dent Res. 2017;20(2):97-104.

22. Freeman $\mathrm{R}$, Maizels J, Wyllie M, Sheiham A. The relationship between health related knowledge, attitudes and dental health behaviours in 14-16-year-old adolescents. Community Dent Health. 1993;10(4):397-404.

23. Xq Q. The third national oral health epidemiological survey report. Beijing: People's medical publishing house; 2008.

24. Hair JF. Multivariate data analysis, 7. Upper Saddle River: Prentice Hall; 2010.

25. Setia MS. Methodology Series Module 5: Sampling Strategies. Indian J Dermatol. 2016;61(5):505-9.

26. Yin W, Yang YM, Chen H, Li X, Wang Z, Cheng L, Yin QD, Fang HZ, Fei W, $\mathrm{Mi} F \mathrm{~L}$, et al. Oral health status in Sichuan Province: findings from the oral health survey of Sichuan, 2015-2016. Int J Oral Sci. 2017;9(1):10-5.

27. World Health Organization. Oral health surveys: basic methods. 5th ed; 2013. p. 44-6.

28. Preisser JS, Stamm JW, Long DL, Kincade ME. Review and recommendations for zero-inflated count regression modeling of dental caries indices in epidemiological studies. Caries Res. 2012;46(4):413-23.

29. Alkhtib A, Ghanim A, Temple-Smith M, Messer LB, Pirotta M, Morgan M. Prevalence of early childhood caries and enamel defects in four and fiveyear old Qatari preschool children. BMC Oral Health. 2016;16(1):73.

30. Wang L, Cheng L, Yuan B, Hong X, Hu T. Association between socioeconomic status and dental caries in elderly people in Sichuan Province, China: a cross-sectional study. BMJ Open. 2017;7(9):e016557.

31. Zhu L, Petersen PE, Wang HY, Bian JY, Zhang BX. Oral health knowledge, attitudes and behaviour of adults in China. Int Dent J. 2005;55(4):231-41.

32. Chankanka O, Levy SM, Marshall TA, Cavanaugh JE, Warren JJ, Broffitt B, Kolker JL. The associations between dietary intakes from 36 to 60 months of age and primary dentition non-cavitated caries and cavitated caries. J Public Health Dent. 2015;75(4):265-73.

33. Anil S, Anand PS. Early Childhood Caries: Prevalence, Risk Factors, and Prevention. Front Pediatr. 2017:5:157.

34. Prakash $P$, Subramaniam $P$, Durgesh BH, Konde S. Prevalence of early childhood caries and associated risk factors in preschool children of urban Bangalore, India: A cross-sectional study. Eur J Dent. 2012;6(2): 141-52.

35. Hooper D, Coughlan J, Mullen M. Structural equation modelling: guidelines for determining model fit. Electron J Bus Res Methods. 2008;6:53-60. 
36. Sheiham A, James WP. A new understanding of the relationship between sugars, dental caries and fluoride use: implications for limits on sugars consumption. Public Health Nutr. 2014;17(10):2176-84.

37. Chen KJ, Gao SS, Duangthip D, Li SKY, Lo ECM, Chu CH. Dental caries status and its associated factors among 5-year-old Hong Kong children: a crosssectional study. BMC Oral Health. 2017;17(1):121.

38. Joybell C, Krishnan R, SK V. Comparison of Two Brushing Methods- Fone's vs Modified Bass Method in Visually Impaired Children Using the Audio Tactile Performance (ATP) Technique. J Clin Diagn Res. 2015;9(3):Zc19-22.

39. Public Health England. Delivering Better Oral Health: An evidence based toolkit for prevention. 3rd ed. London: Public Health England; 2014

40. Qiu RM, Wong MC, Lo EC, Lin HC. Relationship between children's oral health-related behaviors and their caregiver's sense of coherence. BMC Public Health. 2013;13:239.

41. Listl S. Income-related inequalities in dental service utilization by Europeans aged 50+. J Dent Res. 2011;90(6):717-23.

42. Rebelo MA, Cardoso EM, Robinson PG, Vettore MV. Demographics, social position, dental status and oral health-related quality of life in communitydwelling older adults. Qual Life Res. 2016;25(7):1735-42.

43. Kline R. Principles and practice of structural equation modeling. New York: Guilford Press; 2016.

Ready to submit your research? Choose BMC and benefit from:

- fast, convenient online submission

- thorough peer review by experienced researchers in your field

- rapid publication on acceptance

- support for research data, including large and complex data types

- gold Open Access which fosters wider collaboration and increased citations

- maximum visibility for your research: over $100 \mathrm{M}$ website views per year

At $\mathrm{BMC}$, research is always in progress.

Learn more biomedcentral.com/submissions 